PROCEEDINGS OF THE

AMERICAN MATHEMATICAL SOCIETY

Volume 128, Number 7, Pages 2129-2135

S 0002-9939(00)05227-8

Article electronically published on February 23, 2000

\title{
NONSTANDARD SOLVABILITY FOR LINEAR OPERATORS BETWEEN SECTIONS OF VECTOR BUNDLES
}

\author{
HIROSHI AKIYAMA
}

(Communicated by Carl G. Jockusch, Jr.)

\begin{abstract}
Given a certain kind of linear operator $A$ (possibly a differential operator or a properly supported pseudodifferential operator) between sections of Hermitian vector bundles over a Riemannian manifold, a necessary and sufficient condition is obtained for the operator $A$ to be solvable in a class of nonstandard sections in a generalized sense of weak solutions. The existence of a fundamental-solution-like internal section is established in the solvable case.
\end{abstract}

\section{INTRODUCTION}

Let $E$ and $F$ be $C^{\infty}$ Hermitian vector bundles over a Riemannian manifold $M$, and let $A: \Gamma_{0}^{\infty}(E) \rightarrow \Gamma^{\infty}(F)$ be a $\mathbb{C}$-linear operator from the space $\Gamma_{0}^{\infty}(E)$ of $C^{\infty}$ sections of $E$ with compact support to the space $\Gamma^{\infty}(F)$ of $C^{\infty}$ sections of $F$, with formal adjoint $A^{\#}: \Gamma_{0}^{\infty}(F) \rightarrow \Gamma^{\infty}(E)$ such that $A^{\#} \varphi$ is an $L^{2}$-section of $E$ for every $\varphi \in \Gamma_{0}^{\infty}(F)$ (\$3 (3.1)). (For example, the operator $A$ may be a linear differential operator or a properly supported pseudodifferential operator.) Let $T$ be a generalized section of $F$ (see \$2] or [2]).

Consider the equation

$$
A u=T
$$

in the sense that

$$
\left(u, A^{\#} \varphi\right)_{E}=(T, \varphi)_{F} \quad \text { for all } \quad \varphi \in \Gamma_{0}^{\infty}(F) .
$$

Here $(T, \varphi)_{F}$ is defined by extending the inner product $(,)_{F}$ in the space of $L^{2}$ sections of $F$ (4, (4.1)). The left-hand side of the equation in (1.2) can be defined if $u$ is an $L^{2}$-section of $E$, or if $u$ is a generalized section of $E$ and if the support of $A^{\#} \varphi$ is compact for every $\varphi \in \Gamma_{0}^{\infty}(F)$. Then for the equation (1.1) to be solvable, it is clearly necessary that the following condition be satisfied:

$$
\forall \varphi \in \Gamma_{0}^{\infty}(F) \quad\left[A^{\#} \varphi=0 \Longrightarrow(T, \varphi)_{F}=0\right] .
$$

We generalize the notion of solvability in order to be able to deal with nonstandard sections by reformulating (1.1) and (1.2) properly using nonstandard analysis.

Received by the editors March 30, 1998 and, in revised form, September 4, 1998.

1991 Mathematics Subject Classification. Primary 46S20, 03H05, 35D05, 47B38; Secondary 46F10, 47A50, 58G99.

Key words and phrases. Nonstandard analysis, nonstandard extension, transfer principle, saturation principle, hyperfinite-dimensional internal vector space, Hermitian vector bundle, generalized section. 
We then show that the condition $(\mathrm{C} 1)$ is necessary and sufficient for (the reformulated equation of) (1.1) to have a solution in the nonstandard extension $*\left(\Gamma^{\infty}(E)\right)$ of $\Gamma^{\infty}(E)$ (\$4 Theorem 4.2). Our present work is partly motivated by some results in T. Todorov [6] and [7], where existence results are established for linear partial differential equations (with smooth coefficients) on open sets of $\mathbb{R}^{d}$ (in other words, he studies in the product complex line bundle case $E=F=\Omega \times \mathbb{C}$ with $M=\Omega$ an open set of $\mathbb{R}^{d}$ ).

In this paper, we first show that for the nonstandard extension ${ }^{*} A$ of the operator $A$ and for each internal ${ }^{*} C^{\infty}$ section $f$ of the nonstandard extension ${ }^{*} F$ of $F$ (that is, $\left.f \in{ }^{*}\left(\Gamma^{\infty}(F)\right)\right)$, the equation

$$
{ }^{*} A u=f
$$

is solvable in a certain generalized sense of weak solutions if and only if $f$ satisfies a condition similar to $(\mathrm{C} 1)$ (with $T$ replaced by $f$ ) (\$3, Theorem 3.1). Then we show that this treatment applies to the equation (1.1); moreover, we also obtain a result on the existence of a fundamental-solution-like internal section for (1.1) (\&4. Theorem 4.2). Finally we prove the existence of an internal section (depending on $A$ ) that plays a fundamental role in constructing a solution of (1.1) for each generalized section $T$ of $F$ satisfying (C1) (Theorem 4.3) .

We obtain our results using the notion of hyperfinite-dimensional internal vector space in the framework of nonstandard analysis. As for the preliminary knowledge of nonstandard analysis, see for example [3], [4]. We work with a sufficiently saturated nonstandard model.

\section{Notational PRELiminaries}

Let $\mathbb{N}$ be the set of all (strictly) positive integers, and let $\mathbb{R}[$ resp. $\mathbb{C}]$ be the set of all real numbers [resp. complex numbers]. We denote by ${ }^{*} \mathbb{N}_{\infty}:={ }^{*} \mathbb{N} \backslash \mathbb{N}$ the infinite elements in the nonstandard extension ${ }^{*} \mathbb{N}$ of $\mathbb{N}$.

Given a complex vector bundle $\pi_{E}: E \rightarrow M$, we write $E_{x}:=\pi_{E}^{-1}(x)$, the fiber of $E$ over $x \in M$. Let $\Gamma^{\infty}(E)$ denote the space of all $C^{\infty}$ sections of $E$ and $\Gamma_{0}^{\infty}(E)$ the space of $C^{\infty}$ sections of $E$ with compact support. A generalized section of $E$ is defined as a continuous linear functional on the space $\Gamma_{0}^{\infty}\left(E^{\star} \otimes\left|\wedge_{M}\right|\right)$, where $E^{\star}$ is the dual bundle of $E$ and $\left|\wedge_{M}\right|$ the complex line bundle of densities over $M$ (see 2]). The space of generalized sections of $E$ is denoted by $\Gamma^{-\infty}(E)$.

For two vector bundles $E_{1} \rightarrow M_{1}$ and $E_{2} \rightarrow M_{2}$, we denote by $E_{1} \otimes E_{2}$ the vector bundle over $M_{1} \times M_{2}$ such that $\left(E_{1} \otimes E_{2}\right)_{(x, y)}=\left(E_{1}\right)_{x} \otimes\left(E_{2}\right)_{y}$ for every $(x, y) \in$ $M_{1} \times M_{2}$. For simplicity of notation, we write $\otimes$ for ${ }^{*} \otimes$ (the nonstandard extension of $\otimes)$. Similarly, we write $\otimes$ for ${ }^{*} \otimes$.

In what follows, let $(M, g)$ be a (finite-dimensional, $\sigma$-compact) $C^{\infty}$ Riemannian manifold, and let $\pi_{E}: E \rightarrow M$ and $\pi_{F}: F \rightarrow M$ be $C^{\infty}$ Hermitian vector bundles with Hermitian metrics $h_{E}$ and $h_{F}$, respectively. Let $d v_{g} \in \Gamma^{\infty}\left(\left|\wedge_{M}\right|\right)$ be the Riemannian volume density associated with $g$ (see [5]). For $s_{1}, s_{2} \in \Gamma^{\infty}(E)$, we set

$$
\left(s_{1}, s_{2}\right)_{E}=\int_{M} h_{E}\left(s_{1}, s_{2}\right) d v_{g}, \quad\left\|s_{1}\right\|_{E}=\left(s_{1}, s_{1}\right)_{E}^{1 / 2},
$$

if they exist. $\left((,)_{E}\right.$ is extended to be an $L^{2}$-inner product.) Define $(,)_{F}$ and \|\|$_{F}$ in a similar way. For simplicity, we denote by the same notation $(,)_{E}\left[\operatorname{resp} .(,)_{F}\right]$ the nonstandard extension of $(,)_{E}\left[\operatorname{resp} .(,)_{F}\right]$; furthermore, ${ }^{*}\|\|_{E}:={ }^{*}\left(\|\|_{E}\right)$ stands 
for the nonstandard extension of \|\|$_{E}$. The set of standard elements of $*\left(\Gamma_{0}^{\infty}(F)\right)$ is denoted by ${ }^{\sigma}\left(\Gamma_{0}^{\infty}(F)\right)$, that is,

$$
\sigma\left(\Gamma_{0}^{\infty}(F)\right)=\left\{{ }^{*} \varphi: \varphi \in \Gamma_{0}^{\infty}(F)\right\} .
$$

\section{NONSTANDARD SOLVABILITY OF (1.3)}

Let

$$
A: \Gamma_{0}^{\infty}(E) \longrightarrow \Gamma^{\infty}(F)
$$

be a linear operator (a $\mathbb{C}$-linear map) with formal adjoint

$$
A^{\#}: \Gamma_{0}^{\infty}(F) \longrightarrow \Gamma^{\infty}(E)
$$

so that

$$
(A s, \varphi)_{F}=\left(s, A^{\#} \varphi\right)_{E} \in \mathbb{C} \quad\left(s \in \Gamma_{0}^{\infty}(E), \varphi \in \Gamma_{0}^{\infty}(F)\right),
$$

such that

$$
\left.\left\|A^{\#} \varphi\right\|_{E}<\infty \quad \text { (that is, }\left\|A^{\#} \varphi\right\|_{E} \in \mathbb{R}\right) \quad \text { for all } \varphi \in \Gamma_{0}^{\infty}(F) .
$$

By the transfer principle, we have

$$
\left({ }^{*} A \sigma, \zeta\right)_{F}=\left(\sigma,{ }^{*} A^{\#} \zeta\right)_{E} \in{ }^{*} \mathbb{C} \quad\left(\sigma \in{ }^{*}\left(\Gamma_{0}^{\infty}(E)\right), \zeta \in^{*}\left(\Gamma_{0}^{\infty}(F)\right)\right),
$$

where ${ }^{*} A^{\#}:={ }^{*}\left(A^{\#}\right)$.

For $f \in{ }^{*}\left(\Gamma^{\infty}(F)\right)$, we study the existence of an element $u \in{ }^{*}\left(\Gamma^{\infty}(E)\right)$ with ${ }^{*}\|u\|_{E} \in{ }^{*} \mathbb{R}$ that satisfies the equation

$$
{ }^{*} A u=f
$$

in the sense that

$$
\left(u,{ }^{*}\left(A^{\#} \varphi\right)\right)_{E}=\left(f,{ }^{*} \varphi\right)_{F} \quad \text { for all } \quad \varphi \in \Gamma_{0}^{\infty}(F) .
$$

$\left({ }^{*} \varphi\right.$ : the nonstandard extension of $\varphi$.) Note that ${ }^{*}\left(A^{\#} \varphi\right)={ }^{*} A^{\# *} \varphi$ holds for every $\varphi \in \Gamma_{0}^{\infty}(F)$. Note also that in (3.3), since ${ }^{*}\left\|^{*}\left(A^{\#} \varphi\right)\right\|_{E}=\left\|A^{\#} \varphi\right\|_{E} \in \mathbb{R}$ by (3.1), ${ }^{*}\|u\|_{E} \in{ }^{*} \mathbb{R}$ implies that $\left(u,{ }^{*}\left(A^{\#} \varphi\right)\right)_{E} \in{ }^{*} \mathbb{C}$ by transfer of the Schwarz inequality.

The following theorem concerns the solvability of (3.2).

Theorem 3.1. Let $f \in{ }^{*}\left(\Gamma^{\infty}(F)\right)$. Then the equation (3.2) in the sense of (3.3) has a solution $u \in{ }^{*}\left(\Gamma^{\infty}(E)\right)$ with ${ }^{*}\|u\|_{E} \in{ }^{*} \mathbb{R}$ if and only if $f$ satisfies the following condition (C2):

$$
\forall \varphi \in \Gamma_{0}^{\infty}(F) \quad\left[A^{\#} \varphi=0 \Longrightarrow\left(f,{ }^{*} \varphi\right)_{F}=0\right] .
$$

Proof. Suppose an element $u \in{ }^{*}\left(\Gamma^{\infty}(E)\right)$ satisfies ${ }^{*}\|u\|_{E} \in{ }^{*} \mathbb{R}$ and (3.3). Then clearly $f$ satisfies (C2).

Conversely, suppose $f$ satisfies $(\mathrm{C} 2)$. In the case $A^{\#}=0$, we have only to let $u=0 ;$ so assume $A^{\#} \neq 0$. Set

$$
W=\left\{\zeta \in{ }^{*}\left(\Gamma_{0}^{\infty}(F)\right):{ }^{*} A^{\#} \zeta=0 \Longrightarrow(f, \zeta)_{F}=0\right\} .
$$

This is an internal vector space over ${ }^{*} \mathbb{C}$. The set ${ }^{\sigma}\left(\Gamma_{0}^{\infty}(F)\right)$ of standard elements of ${ }^{*}\left(\Gamma_{0}^{\infty}(F)\right)$ is an external subset of $W$; note that ${ }^{*} A^{\# *} \varphi=0$ holds if and only if $A^{\#} \varphi=0$. By the saturation principle, there exists a hyperfinite-dimensional internal vector subspace $V$ of $W$ such that ${ }^{\sigma}\left(\Gamma_{0}^{\infty}(F)\right)$ is a subset of $V$. Let the 
*-dimension of $V$ be ${ }^{*} \operatorname{dim} V=\nu$, which is an infinite hypernatural number $(\nu \in$ $\left.* \mathbb{N}_{\infty}\right)$. We can pick a basis $\left\{\psi_{1}, \ldots, \psi_{\nu}\right\}$ for $V$ such that

$$
\begin{aligned}
& \left(\psi_{i}, \psi_{j}\right)_{F}=\delta_{i j} \quad(i, j=1, \ldots, \nu), \\
& \left\{{ }^{*} A^{\#} \psi_{1}, \ldots,{ }^{*} A^{\#} \psi_{\nu_{0}}\right\} \text { is a basis for }{ }^{*} A^{\#}(V)=\left\{{ }^{*} A^{\#} \zeta: \zeta \in V\right\}, \\
& { }^{*} A^{\#} \psi_{j}=0 \text { for } j=\nu_{0}+1, \ldots, \nu .
\end{aligned}
$$

By applying the transfer principle to (3.1), we have ${ }^{*}\left\|^{*} A^{\#} \psi_{i}\right\|_{E} \in{ }^{*} \mathbb{R}$ for $i=$ $1, \ldots, \nu_{0}$. Therefore

$$
b_{i j}:=\left({ }^{*} A^{\#} \psi_{i},{ }^{*} A^{\#} \psi_{j}\right)_{E} \in{ }^{*} \mathbb{C} \quad\left(i, j=1, \ldots, \nu_{0}\right)
$$

by transfer of the Schwarz inequality, and the $\nu_{0} \times \nu_{0}$ internal Hermitian matrix $B=\left(b_{i j}\right)\left(i, j=1, \ldots, \nu_{0}\right)$ is nonsingular by (3.6).

Let $C=\left(c_{i j}\right)$ be the inverse matrix of $B$. Define $u_{i} \in{ }^{*}\left(\Gamma^{\infty}(E)\right)\left(i=1, \ldots, \nu_{0}\right)$ by the internal sum

$$
u_{i}=\sum_{k=1}^{\nu_{0}} c_{i k}{ }^{*} A^{\#} \psi_{k}
$$

Then for $i, j=1, \ldots, \nu_{0}$, it holds that

$$
\left(u_{i},{ }^{*} A^{\#} \psi_{j}\right)_{E}=\delta_{i j} \quad(\text { Kronecker delta }), \quad\left(u_{i}, u_{j}\right)_{E}=c_{i j} .
$$

Thus

$$
u=\sum_{i=1}^{\nu_{0}}\left(f, \psi_{i}\right)_{F} u_{i}
$$

satisfies ${ }^{*}\|u\|_{E} \in{ }^{*} \mathbb{R}$ and

$$
\left(u,{ }^{*} A^{\#} \psi_{j}\right)_{E}=\left(f, \psi_{j}\right)_{F} \quad(j=1, \ldots, \nu) .
$$

(Note that both sides of (3.10) are 0 for $j=\nu_{0}+1, \ldots, \nu$.) Since every ${ }^{*} \varphi \in$ $\sigma\left(\Gamma_{0}^{\infty}(F)\right)$ is expressed in the form

$$
{ }^{*} \varphi=\sum_{j=1}^{\nu} \alpha_{j} \psi_{j} \quad\left(\alpha_{j} \in{ }^{*} \mathbb{C}\right),
$$

it follows from (3.10) that the above $u$ in (3.9) satisfies (3.3).

Remark 3.1. From the above proof, we see that if $f \in \epsilon^{*}\left(\Gamma^{\infty}(F)\right)$ satisfies (C2) and if the support of $A^{\#} \varphi$ is compact for every $\varphi \in \Gamma_{0}^{\infty}(F)$, then it holds that ${ }^{*} A^{\#} \psi_{j} \in{ }^{*}\left(\Gamma_{0}^{\infty}(E)\right)\left(j=1, \ldots, \nu_{0}\right)$ and thus we can choose $u$ in Theorem 3.1 in such a way that $u \in{ }^{*}\left(\Gamma_{0}^{\infty}(E)\right)$.

Remark 3.2. The operator $A$ admits a $*$-integral representation with ${ }^{*} C^{\infty}$ kernel ([1, Proposition 2.2]).

\section{NONSTANDARD SOlVABILITY OF (1.1)}

For a section $\varphi \in \Gamma_{0}^{\infty}(F)$, define ${ }^{h} \varphi \in \Gamma_{0}^{\infty}\left(F^{\star}\right)$ by

$$
{ }^{h} \varphi=h_{F}(\cdot, \varphi) .
$$

For a generalized section $T \in \Gamma^{-\infty}(F)$ and a section $\varphi \in \Gamma_{0}^{\infty}(F)$, put

$$
(T, \varphi)_{F}=T\left({ }^{h} \varphi \otimes d v_{g}\right) \in \mathbb{C} .
$$


Note that this is consistent with the notation $(,)_{F}$ in 2.1) if $T \in \Gamma^{\infty}(F)(\subset$ $\left.\Gamma^{-\infty}(F)\right)$.

Using the nonstandard extension ${ }^{*} h_{F}$ of $h_{F}$, define, for $\zeta \in{ }^{*}\left(\Gamma_{0}^{\infty}(F)\right)$,

$$
{ }^{h} \zeta={ }^{*} h_{F}(\cdot, \zeta) \in{ }^{*}\left(\Gamma_{0}^{\infty}\left(F^{\star}\right)\right),
$$

so that ${ }^{h} \zeta \otimes{ }^{*} d v_{g} \in{ }^{*}\left(\Gamma_{0}^{\infty}\left(F^{\star} \otimes\left|\wedge_{M}\right|\right)\right)$. Then for the nonstandard extension ${ }^{*} T$ of $T \in \Gamma^{-\infty}(F)$, we have

$$
\left({ }^{*} T, \zeta\right)_{F}={ }^{*} T\left({ }^{h} \zeta \otimes * d v_{g}\right) \in{ }^{*} \mathbb{C} .
$$

The following key lemma enables us to apply Theorem 3.1 to the study of the equation (1.1).

Lemma 4.1 (Key Lemma). There exists a $\mathbb{C}$-linear injection

$$
\beta: \Gamma^{-\infty}(F) \longrightarrow{ }^{*}\left(\Gamma_{0}^{\infty}(F)\right)
$$

such that for each $T \in \Gamma^{-\infty}(F), \beta(T)$ satisfies

$$
\left(\beta(T),{ }^{*} \varphi\right)_{F}=(T, \varphi)_{F} \quad\left(\varphi \in \Gamma_{0}^{\infty}(F)\right) .
$$

Proof (cf. [1, Theorem 2.3]). Consider ${ }^{*}\left(\Gamma_{0}^{\infty}(F)\right)$ instead of $W$ given in (3.4) and proceed as in the proof of Theorem 3.1 to obtain a hyperfinite-dimensional internal vector subspace $V$ of ${ }^{*}\left(\Gamma_{0}^{\infty}(F)\right)$ such that ${ }^{\sigma}\left(\Gamma_{0}^{\infty}(F)\right)$ is an external subset of $V$. Pick a basis $\left\{\psi_{1}, \ldots, \psi_{\nu}\right\}$ (where ${ }^{*} \operatorname{dim} V=\nu \in{ }^{*} \mathbb{N}_{\infty}$ ) for $V$ satisfying (3.5), (3.6), and (3.7). (We need (3.6) and (3.7) for later use in the proof of Theorem 4.3, but not here.) Define a $\mathbb{C}$-linear map $\beta: \Gamma^{-\infty}(F) \longrightarrow *\left(\Gamma_{0}^{\infty}(F)\right)$ by

$$
\beta(T)=\sum_{i=1}^{\nu}\left({ }^{*} T, \psi_{i}\right)_{F} \psi_{i} \quad\left(T \in \Gamma^{-\infty}(F)\right) .
$$

Then

$$
\left(\beta(T), \psi_{j}\right)_{F}=\left({ }^{*} T, \psi_{j}\right)_{F} \quad \text { for } \quad j=1, \ldots, \nu .
$$

Since each ${ }^{*} \varphi \in{ }^{\sigma}\left(\Gamma_{0}^{\infty}(F)\right)$ is expressed in the form (3.11), we see that

$$
\left(\beta(T),{ }^{*} \varphi\right)_{F}=\left({ }^{*} T,{ }^{*} \varphi\right)_{F}={ }^{*}\left((T, \varphi)_{F}\right)=(T, \varphi)_{F} .
$$

The injectivity of $\beta$ is clear.

Now we reformulate (1.1) and (1.2). The following theorem concerns not only the nonstandard solvability of (1.1) but also the existence of a related fundamentalsolution-like internal section.

Theorem 4.2. Let $A: \Gamma_{0}^{\infty}(E) \longrightarrow \Gamma^{\infty}(F)$ be as in $\S 3$.

(a) Let $T \in \Gamma^{-\infty}(F)$, and consider the equation

$$
{ }^{*} A u={ }^{*} T
$$

in the sense that

$$
\left(u,{ }^{*}\left(A^{\#} \varphi\right)\right)_{E}=\left({ }^{*} T,{ }^{*} \varphi\right)_{F}\left(=(T, \varphi)_{F}\right) \quad \text { for all } \varphi \in \Gamma_{0}^{\infty}(F) .
$$

Then (4.3) has a solution $u \in{ }^{*}\left(\Gamma^{\infty}(E)\right)$ with $*\|u\|_{E} \in{ }^{*} \mathbb{R}$ if and only if $T$ satisfies the condition $(\mathrm{C} 1)$. 
(b) There exists an internal ${ }^{*} C^{\infty}$ section

$$
G \in{ }^{*}\left(\Gamma^{\infty}\left(E \otimes F^{\star}\right)\right)
$$

such that, for each $x \in{ }^{*} M$, the internal support of $G(x, \cdot)$ is *-compact and such that, for $T \in \Gamma^{-\infty}(F)$ satisfying the condition (C1), the *-integral

$$
u(x)=\int_{{ }^{M} M} G(x, y) \beta(T)(y)^{*} d v_{g}(y) \quad\left(x \in{ }^{*} M\right)
$$

gives a solution of (4.3) in the sense of (4.4).

Proof. To prove (a), let $T \in \Gamma^{-\infty}(F)$. By (4.2), $\beta(T)$ satisfies the condition (C2) (with $f$ replaced by $\beta(T)$ ) if and only if $T$ satisfies the condition (C1). Then (a) follows from Theorem 3.1 and Lemma 4.1

To prove (b), observe that, for $T \in \Gamma^{-\infty}(F)$ satisfying the condition (C1), the internal set

$$
W_{T}:=\left\{\zeta \in{ }^{*}\left(\Gamma_{0}^{\infty}(F)\right):{ }^{*} A^{\#} \zeta=0 \Longrightarrow\left({ }^{*} T, \zeta\right)_{F}=0\right\}
$$

coincides with $*\left(\Gamma_{0}^{\infty}(F)\right)$ by the transfer principle. Consequently, $W_{T}$ does not depend on the choice of $T$ satisfying $(\mathrm{C} 1)$. We proceed as in the proof of Lemma 4.1. Let $\psi_{i}(i=1, \ldots, \nu)$ be as in the proof of Lemma 4.1, and using these, define $u_{i}\left(i=1, \ldots, \nu_{0}\right)$ as in (3.8). Define $G \in{ }^{*}\left(\Gamma^{\infty}\left(E \otimes F^{\star}\right)\right)$ by

$$
G(x, y) \gamma(y)=\sum_{i=1}^{\nu_{0}}{ }^{*} h_{F}\left(\gamma(y), \psi_{i}(y)\right) u_{i}(x) \quad\left(x, y \in{ }^{*} M\right)
$$

for all $\gamma \in{ }^{*}\left(\Gamma^{\infty}(F)\right)$. Then the internal support of $G(x, \cdot)$ is $*$-compact for each $x \in{ }^{*} M$. Furthermore, $u$ defined by the formula (3.9) with $f$ replaced by $\beta(T)$ is a solution of (4.3) in the sense of (4.4) and is represented as (4.5).

Remark 4.1. Let $u \in{ }^{*}\left(\Gamma^{\infty}(E)\right)$ be a solution of (4.3) with ${ }^{*}\|u\|_{E} \in{ }^{*} \mathbb{R}$.

(1) If $u$ happens to be standard, so that $u={ }^{*} v$ for some $v \in \Gamma^{\infty}(E)$ with $\|v\|_{E} \in \mathbb{R}$ (that is, $\|v\|_{E}<\infty$ ), then, by (4.4),

$$
\left(v, A^{\#} \varphi\right)_{E}=(T, \varphi)_{F} \quad \text { for all } \varphi \in \Gamma_{0}^{\infty}(F) .
$$

In this sense, we have $A v=T$.

(2) Consider the case where the support of $A^{\#} \varphi$ is compact for every $\varphi \in \Gamma_{0}^{\infty}(F)$. If there exists a generalized section $U \in \Gamma^{-\infty}(E)$ such that

$$
\left(u,{ }^{*} s\right)_{E}=(U, s)_{E} \quad \text { for all } s \in \Gamma_{0}^{\infty}(E),
$$

then, by (4.4),

$$
\left(U, A^{\#} \varphi\right)_{E}=(T, \varphi)_{F} \quad \text { for all } \quad \varphi \in \Gamma_{0}^{\infty}(F) .
$$

In this sense, we have $A U=T$.

Finally, we have the following.

Theorem 4.3. Let $A: \Gamma_{0}^{\infty}(E) \longrightarrow \Gamma^{\infty}(F)$ be as in $\S 3$. Then there exists an internal ${ }^{*} C^{\infty}$ section

$$
K \in^{*}\left(\Gamma^{\infty}\left(E \otimes\left(F^{\star} \otimes\left|\wedge_{M}\right|\right)\right)\right)
$$

with the following properties:

(a) For each $x \in{ }^{*} M$, the internal support of $K(x, \cdot)$ is $*$-compact. 
(b) For each generalized section $T \in \Gamma^{-\infty}(F)$ satisfying the condition (C1), the internal section $u \in{ }^{*}\left(\Gamma^{\infty}(E)\right)$ defined by

$$
u(x)={ }^{*} T(K(x, \cdot)) \quad\left(x \in{ }^{*} M\right)
$$

satisfies ${ }^{*}\|u\|_{E} \in{ }^{*} \mathbb{R}$ and is a solution of (4.3) in the sense of (4.4). Here ${ }^{*} T$ is extended so as to be ${ }^{*} E_{x}$-valued in a natural manner $\left({ }^{*} E_{x}={ }^{*}\left(\pi_{E}\right)^{-1}(x)\right)$.

Proof. Let $\psi_{i}(i=1, \ldots, \nu)$ and $u_{i}\left(i=1, \ldots, \nu_{0}\right)$ be as in the proof of Theorem 4.2. We define

$$
K \in{ }^{*}\left(\Gamma^{\infty}\left(E \otimes\left(F^{\star} \otimes\left|\wedge_{M}\right|\right)\right)\right)
$$

by

$$
K(x, y)=\sum_{i=1}^{\nu_{0}} u_{i}(x) \otimes\left({ }^{h} \psi_{i} \otimes^{*} d v_{g}\right)(y) \quad\left(x, y \in{ }^{*} M\right) .
$$

Then the internal support of $K(x, \cdot)$ is $*$-compact for each $x \in{ }^{*} M$. Put

$$
u(x)={ }^{*} T(K(x, \cdot))=\sum_{i=1}^{\nu_{0}}{ }^{*} T\left({ }^{h} \psi_{i} \otimes^{*} d v_{g}\right) u_{i}(x)=\sum_{i=1}^{\nu_{0}}\left({ }^{*} T, \psi_{i}\right)_{F} u_{i}(x)
$$

for $x \in{ }^{*} M$. Then $u \in{ }^{*}\left(\Gamma^{\infty}(E)\right)$ and ${ }^{*}\|u\|_{E} \in{ }^{*} \mathbb{R}$. Moreover, we have

$$
\left(u,{ }^{*} A^{\#} \psi_{j}\right)_{E}=\left({ }^{*} T, \psi_{j}\right)_{F} \quad(j=1, \ldots, \nu) .
$$

(Note that both sides of (4.6) are 0 for $j=\nu_{0}+1, \ldots, \nu$.) Hence $u$ satisfies (4.4) by the expression (3.11) of ${ }^{*} \varphi \in{ }^{\sigma}\left(\Gamma_{0}^{\infty}(F)\right)$.

\section{REFERENCES}

1. H. Akiyama, Nonstandard representations of generalized sections of vector bundles, Osaka J. Math., 32 (1995), 817-822. MR 97a:46113

2. V. Guillemin and S. Sternberg, Geometric Asymptotics, rev. ed., Mathematical surveys and monographs No. 14, Amer. Math. Soc., Providence, R.I., 1990. MR 58:24404

3. A. E. Hurd and P. A. Loeb, An Introduction to Nonstandard Real Analysis, Academic Press, New York, 1985. MR 87d:03184

4. T. Lindstr $\phi \mathrm{m}$, An invitation to nonstandard analysis, in "Nonstandard Analysis and its Applications" (N. Cutland, ed.), London Math. Soc. Student Texts 10, Cambridge Univ. Press, 1988, pp. 1-105. MR 89m:03060

5. S. Sternberg, Lectures on Differential Geometry, 2nd ed., Chelsea, New York, 1983. MR 33:1797 MR 88f:58001

6. T. Todorov, An existence result for a class of partial differential equations with smooth coefficients, in "Advances in Analysis, Probability and Mathematical Physics: Contributions of Nonstandard Analysis" (S. Albeverio, W. A. J. Luxemburg, M. P. H. Wolff, eds.), Math. Appl. Vol. 314, Kluwer Acad. Publ., Dordrecht, 1995, pp. 107-121. MR 96m:35005

7. T. Todorov, An existence result for linear partial differenial equations with $C^{\infty}$ coefficients in an algebra of generalized functions, Trans. Amer. Math. Soc., 348 (1996), 673-689. MR 96m:35004

Department of Applied Mathematics, Faculty of Engineering, Shizuoka University, HAMAMATSU 432-8561, JAPAN

E-mail address: tshakiy@eng.shizuoka.ac.jp 